\title{
Tibiofibular syndesmosis in acute ankle fractures: additional value of an oblique MR image plane
}

\author{
John J. Hermans • Annechien Beumer • \\ Wim C. J. Hop • Adrianus F. C. M. Moonen • \\ Abida Z. Ginai
}

Received: 22 October 2010 /Revised: 24 February 2011 / Accepted: 10 April 2011 /Published online: 30 April 2011

(C) The Author(s) 2011. This article is published with open access at Springerlink.com

\begin{abstract}
Objective To evaluate the additional value of a $45^{\circ}$ oblique MRI scan plane for assessing the anterior and posterior distal tibiofibular syndesmotic ligaments in patients with an acute ankle fracture.

Materials and methods Prospectively, data were collected for 44 consecutive patients with an acute ankle fracture who underwent a radiograph (AP, lateral, and mortise view) as well as an MRI in both the standard three orthogonal planes and in an additional $45^{\circ}$ oblique plane. The fractures on the radiographs were classified according to Lauge-Hansen (LH). The anterior (ATIFL) and posterior (PTIFL) distal tibiofibular ligaments, as well as the presence of a bony avulsion in both the axial and oblique planes was evaluated on MRI. MRI findings regarding syndesmotic injury in the axial and oblique planes were compared to syndesmotic
\end{abstract}

J. J. Hermans $(\bowtie) \cdot$ A. Z. Ginai

Department of Radiology, Erasmus University Medical Center,

PO Box 2040, 3000 CA, Rotterdam, The Netherlands

e-mail: jjhermans@gmail.com

A. Z. Ginai

e-mail: a.ginai@erasmusmc.nl

A. Beumer - A. F. C. M. Moonen

Department of Orthopaedics, Amphia Hospital,

PO Box 90158, 4800 RK, Breda, The Netherlands

A. Beumer

e-mail: achbeumer@hotmail.com

A. F. C. M. Moonen

e-mail: sjorsmoonen@hotmail.com

W. C. J. Hop

Department of Biostatistics, Erasmus University Medical Center, PO Box 2040, 3000 CA, Rotterdam, The Netherlands

e-mail: w.hop@erasmusmc.nl injury predicted by LH. Kappa and the agreement score were calculated to determine the interobserver agreement. The Wilcoxon signed rank test and McNemar's test were used to compare the two scan planes.

Results The interobserver agreement $(\mathrm{K})$ and agreement score [AS (\%)] regarding injury of the ATIFL and PTIFL and the presence of a fibular or tibial avulsion fracture were good to excellent in both the axial and oblique image planes ( $\mathrm{k} 0.61-0.92$, AS 84-95\%). For both ligaments the oblique image plane indicated significantly less injury than the axial plane $(p<0.001)$. There was no significant difference in detection of an avulsion fracture in the axial or oblique plane, neither anteriorly $(p=0.50)$ nor posteriorly $(p=1.00)$. With syndesmotic injury as predicted by LH as comparison, the specificity in the oblique MR plane increased for both anterior (to $86 \%$ from $7 \%$ ) and posterior (to $86 \%$ from $48 \%$ ) syndesmotic injury when compared to the axial plane.

Conclusion Our results show the additional value of an $45^{\circ}$ oblique MR image plane for detection of injury of the anterior and posterior distal tibiofibular syndesmoses in acute ankle fractures. Findings of syndesmotic injury in the oblique MRI plane were closer to the diagnosis as assumed by the Lauge-Hansen classification than in the axial plane. With more accurate information, the surgeon can better decide when to stabilize syndesmotic injury in acute ankle fractures.

Keywords Ankle · Fracture - Ligament · Tibiofibular syndesmosis $\cdot$ Radiography $\cdot$ MRI

\section{Abbreviations}

ATIFL Anterior tibiofibular ligament

PTIFL Posterior tibiofibular ligament 


\section{Introduction}

Disruption of the distal tibiofibular syndesmosis in ankle fractures is common and usually results from an external rotation injury. In up to $13 \%$ of all ankle fractures and in $20 \%$ of patients requiring internal fixation, there will be an associated injury to the syndesmosis [1-4]. It is commonly agreed that adequate reduction of ankle fractures reduces late osteoarthritis. However, evaluating syndesmotic stability in ankle fractures is still a subject of debate as it is unclear when to stabilize an injured syndesmosis [5-12].

The value of MRI in acute and chronic syndesmotic injuries has been described in several articles, and the axial image is known to be the most useful image plane [13-17]. However, imaging in the axial plane can result in falsepositive findings $[14,18]$ since the anterior and posterior distal tibiofibular ligament run in an oblique plane $[19,20]$. Several studies reported that the most commonly injured and reliably visualized anterior tibiofibular ligament on MRI might not be seen in its entire length on one single transverse image and a partially imaged ligament may be mistaken for a tear despite careful observation of contiguous slices $[16,21,22]$. A recent study demonstrated that the anterior and posterior syndesmotic ligaments are better depicted and that the integrity of the ligaments can be better evaluated in an oblique image plane of about $45^{\circ}$ when compared to the axial plane [23]. Therefore, this prospective study evaluated the additional value of an oblique MRI scan plane for assessing the anterior and posterior distal tibiofibular syndesmotic ligaments in patients with an acute ankle fracture.

\section{Materials and methods}

The data were collected and analyzed from 44 consecutive patients with an acute ankle fracture who underwent a radiograph as well as an MRI in both the standard three orthogonal planes and in an additional oblique plane. In the 44 patients, the fracture distribution according to LaugeHansen was 59\% supination external rotation (SE), 5\% pronation external rotation (PE), 25\% supination adduction (SA), 9\% pronation adduction (PA), and 2\% pronation dorsiflexion (PD). The mean age of the patients was 36 years and ranged from 16 to 61 years; 25 were men and 19 were women. In 24 patients the injury involved the left and in 20 the right ankle. In 16 patients the fracture was treated with an open reduction and internal fixation (ORIF), with a setscrew placed in 4 patients.

Written informed consent was obtained, and the study was approved by the institutional review board. Inclusion criteria were an acute ankle fracture that had occurred within $48 \mathrm{~h}$ and closure of the growth plates. Exclusion criteria were associated neurological or vascular injuries, fractures associated with a hindfoot or midfoot fracture, a former trauma of the ankle, contraindications for MRI, and an insufficient knowledge of the Dutch language.

Radiographs obtained at presentation included AP, lateral, and mortise views. The fractures on the radiographs were classified according to Lauge-Hansen (Table 1) by a radiologist with 11 years experience in musculoskeletal radiology (J.H.).

MRI was performed on a $1.5 \mathrm{~T}$ Gyroscan (Philips, Best, the Netherlands) with a wrap-around ankle coil (E1 coil). The foot was kept in a fixed (neutral) position and stabilized with sandbags or a plaster. Dual TSE images $(\mathrm{TR}=3,500-4,500 \mathrm{~ms} ; \mathrm{TE}=11 \mathrm{~ms}$; $\mathrm{TE}=120 \mathrm{~ms}$; echo train length $=14$ ) were performed in three orthogonal planes, i.e., axial, coronal, and sagittal, and in an additional $45^{\circ}$ oblique plane. The oblique image plane was defined in the coronal and sagittal views (Fig. 1). In the coronal view the $45^{\circ}$ angle of the oblique plane was related to the tibial plafond and ran in a caudal-cranial and lateral-medial direction through the distal fibula. In the sagittal view the direction of the oblique plane ran parallel to a line along the inferior border of the anterior and posterior tibia. All series were performed with a $512 \times 512$ matrix. The field of view was $18-20 \mathrm{~cm}$ for coronal and sagittal imaging, and $12-$ $15 \mathrm{~cm}$ for axial and oblique imaging. The slice thickness of the images was $3.0 \mathrm{~mm}$ (gap $0.3 \mathrm{~mm}$ ) with an NSA of 2. A STIR, i.e., a short tau inversion recovery image (TR = 1,460 ms; TE = $15 \mathrm{~ms}$; echo train length = 14), was performed in the coronal plane, with a field of view of 18 $20 \mathrm{~cm}$, a slice thickness of $4 \mathrm{~mm}$ (gap $0.4 \mathrm{~mm}$ ), and a $256 \times$ 256 matrix.

The MR images were independently analyzed by two radiologists with 11 and 31 years of musculoskeletal experience (J.H., A.G.). They were blinded to the results of the radiographs. The majority of the patients were examined with MRI on the day of injury. All were examined within 10 days of injury, and five patients were examined postoperatively.

The anterior and posterior syndesmosis consist of the ligaments and their tibial and fibular attachment sites. We first evaluated these two aspects of the syndesmosis separately and then combined these findings, as this coincides with clinical practice regarding syndesmotic injury. On MRI the syndesmotic ligaments, i.e., the anterior and posterior distal tibiofibular ligament, were evaluated in both the axial and oblique planes and assigned a score: $0=$ normal, $1=$ thickened, $2=$ partially ruptured, or $3=$ completely ruptured. A normal distal tibiofibular ligament consisted of multiple continuous thin fibers interspersed with normal high signal intensity fat on dual TSE-weighted images. In a thickened ligament the continuous fibers were thickened, not sharp and the signal intensity of fat was 
Table 1 The Lauge-Hansen fracture classification describes the mechanisms of ankle fractures based on the position of the foot at the time of injury and the direction in which the talus moves within the ankle mortise. It defines both the bony and ligamentous injury.
The following five groups of ankle fractures can be discerned: supination adduction, supination eversion, pronation abduction, pronation eversion, and pronation dorsiflexion. Depending on the degree of severity, the main groups can be further divided into stages

Type of injury (foot position/direction of Pathology

force)

Supination adduction

Supination eversion (external rotation)

Pronation abduction

Pronation eversion (external rotation)

Pronation dorsiflexion/pilon fracture
1) Transverse fracture fibula at or distal to the level of the tibiofibular joint/tear of collateral ligaments

2) Vertical, oblique fracture of the medial malleolus/tear of the deltoid ligament

1) Disruption of the anterior tibiofibular ligament or an avulsion of its tibial attachment (Tillaux fracture) or fibular attachment

2) Spiral, oblique fracture of the distal fibula. The fracture line runs from proximal posterior to distal anterior at a variable distance from the tibiotalar joint

3) Disruption of the posterior tibiofibular ligament or fracture of the posterior malleolus

4) Fracture of the medial malleolus or rupture of the deltoid ligament

1) Transverse fracture of the medial malleolus or rupture of the deltoid ligament

2) Rupture of the anterior and posterior syndesmotic ligaments or avulsion fracture of their insertion(s)

3) Short, oblique fracture of the fibula $0.5-1 \mathrm{~cm}$ above the distal articular surface of the tibia

1) Transverse fracture of the medial malleolus or disruption of the deltoid ligament

2) Disruption of the anterior tibiofibular ligament. The ligament may avulse its tibial attachment (Tillaux fracture)

3) High oblique, spiral fibular fracture. No fracture is less than $2.5 \mathrm{~cm}$ above the tibiotalar joint. The fracture pattern runs from proximal anterior to distal posterior. The fibula may fracture proximally at the neck (Maisonneuve fracture)

4) Rupture of posterior tibiofibular ligament or avulsion fracture of the posterolateral tibia

1) Fracture of the medial malleolus

2) Fracture of the anterior margin of the tibia

3) Supramalleolar fracture of the fibula

4) Transverse fracture of the posterior tibial surface intermediate on dual TSE-weighted images. A complete rupture was defined when the ligament was either discontinuous or invisible or showed increased signal intensity with fluid in the ligament on T2-weighted TSE images. In a partially ruptured ligament the discontinuity was not complete $[16,18,20,23,24]$. The presence of a bony avulsion, both anteriorly and posteriorly, was also assigned a score: $0=$ no avulsion, $1=$ tibial avulsion, $2=$ fibular avulsion, $3=$ avulsion of both tibial and fibular tubercle. Anteriorly this classification was according to Wagstaffe. Posteriorly a fracture of the malleolus tertius, irrespective of its size, was defined as an avulsion.
Fig. 1 Coronal (a) and sagittal (b) MR images, which indicate the $45^{\circ}$ oblique image plane for the anterior and posterior distal tibiofibular ligament. In the coronal plane, the angle of $45^{\circ}$ is related to the tibial plafond. In the sagittal view, the direction of the oblique plane runs parallel to a line along the inferior border of the anterior and posterior tibia
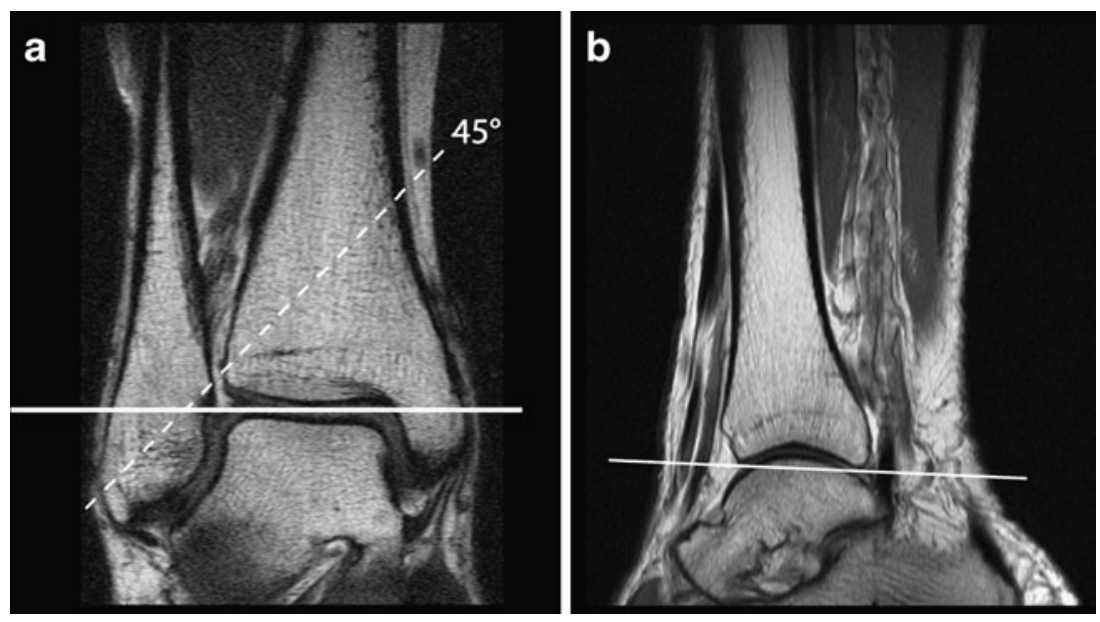
The findings of the distal tibiofibular ligament in combination with the presence of a bony avulsion were combined into the overall presence of anterior or posterior syndesmotic injury: $0=$ normal syndesmosis, i.e., normal ligament without bony avulsion; $1=$ thickened syndesmosis, i.e., thickened ligament without bony avulsion; $2=$ partially ruptured syndesmosis, i.e., partially ruptured ligament without bony avulsion; $3=$ completely ruptured syndesmosis, i.e., ruptured ligament without a bony avulsion or an intact ligament with a bony avulsion.

The interobserver agreement was determined by the kappa score $(\kappa)$ (poor agreement $0.00-0.20$, fair $0.21-0.40$, moderate $0.41-0.60$, good $0.61-0.80$, very good $0.81-0.90$, excellent $0.91-1.00$ ). In case of ordinal scores, i.e., the aspect of the ATIFL and PTIFL, a weighted kappa score was determined. The Wilcoxon signed rank test was used to compare the axial and oblique scan planes regarding the aspect of the ATIFL and PTIFL, and the overall injury scores of anterior and posterior syndesmotic injury. The McNemar test was used to compare the axial and oblique scan planes regarding the presence of an avulsion fracture. The sensitivity and specificity for detection of syndesmotic injury were determined when MRI findings were compared with Lauge-Hansen. A two-sided $p$-value less than 0.05 was considered as significant. Statistical analysis was performed with Stat (version 11.0; Statacorp., College Station, TX, USA).

\section{Results}

The $k$ values regarding the aspect of the ATIFL were 0.61 and 0.92 for the axial and oblique image planes, respectively, with AS of 84 and 91\% (Table 2). The $\mathrm{k}$ scores regarding the presence of a tibial or fibular avulsion fracture of the ATIFL were 0.71 and 0.84 for the axial and oblique image planes, respectively, with AS of 91 and $95 \%$.

Compared to the axial plane, the oblique plane demonstrated lower injury scores in $45 \%(20 / 44)$ and equal injury scores in 55\% (24/44) for the ATIFL $(p<0.001)$ (Fig. 2). Nine patients showed an avulsion fracture anteriorly. The presence of a tibial or fibular avulsion of the ATIFL was not significantly different in the axial and oblique planes ( $p=$ 0.50 ). Anteriorly, $2 / 3$ patients with a tibial and $5 / 6$ patients with a fibular avulsion fracture showed injury of the ATIFL in the axial plane, whereas in the oblique plane none of the avulsion fractures were associated with injury of the ATIFL (Figs. 3, 4).

The kappa for the aspect of the PTIFL was 0.83 and 0.86 for the axial and oblique image planes, respectively, with AS of 84 and $91 \%$ (Table 2). The kappa regarding the presence of a tibial or fibular avulsion fracture of the PTIFL was 0.83 for both the axial and oblique image planes, with an AS of $93 \%$ for both planes.

Compared to the axial plane, the oblique plane demonstrated lower injury scores in 62\% (27/44) and equal injury scores in 36\% (16/44) for the PTIFL $(p<0.001)$. In $2 \%(1 /$ $44)$, the injury score in the oblique plane was higher than in the axial plane. Eleven patients showed an avulsion fracture posteriorly. The presence of a tibial or fibular avulsion of the PTIFL was not significantly different in the axial and oblique planes $(p=1.00)$. Posteriorly there were only avulsion fractures of the tibia and none of the fibula. In 6/ 11 patients with a posterior tibial avulsion, the PTIFL was injured in the axial plane but not in the oblique plane (Fig. 5)

Findings of ligament integrity and the presence of an avulsion fracture were combined to determine overall anterior or posterior syndesmotic injury. The kappa regarding anterior syndesmotic injury was 0.48 and 0.81 in the axial and oblique image planes, respectively, with AS of 84 and $86 \%$. The oblique image plane demonstrated significantly less anterior syndesmotic injury than the axial plane $(p<0.001)$. Regarding posterior syndesmotic injury, the kappa was 0.76 and 0.79 for the axial and oblique image planes, respectively, with an AS of 75 and $79 \%$. The oblique image plane showed significantly less posterior syndesmotic injury than the axial plane $(p<0.001)$.
Table 2 Interobserver agreement ( $\mathrm{k}$ ) and agreement score [AS (\%)] between the axial and oblique MRI planes regarding the aspect of the anterior (ATIFL) and posterior (PTIFL) distal tibiofibular ligaments, the presence of a tibial or fibular avulsion fracture, and overall presence of anterior or posterior syndesmotic injury. $P$-values refer to comparison between the axial and oblique planes

\begin{tabular}{|c|c|c|c|c|c|c|c|c|c|c|}
\hline & \multicolumn{5}{|c|}{ ATIFL } & \multicolumn{5}{|c|}{ PTIFL } \\
\hline & \multicolumn{2}{|l|}{ Axial } & \multicolumn{2}{|c|}{ Oblique } & \multirow[t]{2}{*}{$p$} & \multicolumn{2}{|l|}{ Axial } & \multicolumn{2}{|c|}{ Oblique } & \multirow[t]{2}{*}{$p$} \\
\hline & K & AS (\%) & K & AS (\%) & & K & AS (\%) & K & AS (\%) & \\
\hline Ligament & 0.61 & 84 & 0.92 & 91 & $<0.001$ & 0.83 & 84 & 0.86 & 91 & $<0.001$ \\
\hline Avulsion & 0.71 & 91 & 0.84 & 95 & 0.50 & 0.83 & 93 & 0.83 & 91 & 1.00 \\
\hline Syndesmosis & 0.48 & 84 & 0.81 & 86 & $<0.001$ & 0.79 & 80 & 0.83 & 89 & $<0.001$ \\
\hline
\end{tabular}


Fig. 2 Axial (a) and oblique (b) proton density TSE (turbo spin echo) images. In the axial plane (a), the multifascicular ATIFL (l) is discontinuous, while in the oblique plane (b) the fascicles of the ATIFL are intact. In both planes the PTIFL (2) is intact. $T$ Tibia, $F$ fibula, Ant anterior
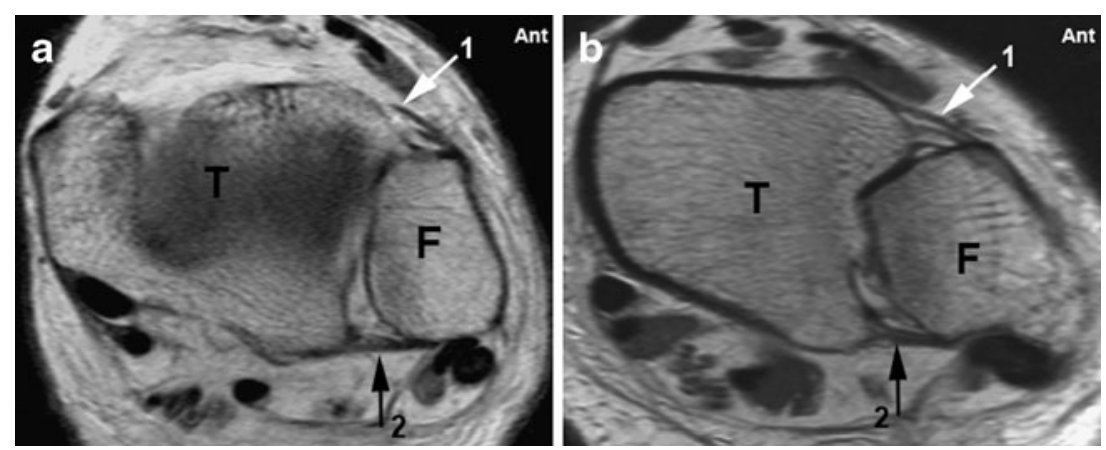

We compared the overall anterior and posterior syndesmotic injury as found on MRI with syndesmotic injury as defined by the Lauge-Hansen fracture classification on the radiograph (Table 3). In 14 patients, LaugeHansen predicted absence of injury of the anterior syndesmosis, whereas MRI demonstrated absence of injury in 1 patient in the axial plane and in 12/14 patients in the oblique plane. In 30 patients Lauge-Hansen predicted presence of injury of the anterior syndesmosis, which was shown in 30 and 28 patients in the axial and oblique planes, respectively (Table 3 ). Compared to Lauge-Hansen, this resulted in a sensitivity and specificity of 100 and $7 \%$, respectively, for the axial plane, and of 93 and $86 \%$ for the oblique plane for detection of anterior syndesmotic injury.

In 29 patients Lauge-Hansen predicted absence of injury of the posterior syndesmosis, whereas MRI demonstrated absence of injury in 14 patients in the axial plane and in 25 patients in the oblique plane (Fig. 6). In 15 patients LaugeHansen predicted presence of injury of the posterior syndesmosis, which was shown in 13 and 11 patients in the axial and oblique planes, respectively (Table 3). Compared to $\mathrm{LH}$, this resulted in a sensitivity and specificity of 87 and $48 \%$, respectively, for the axial planes, and of 73 and $86 \%$ for the oblique plane for detection of posterior syndesmotic injury.

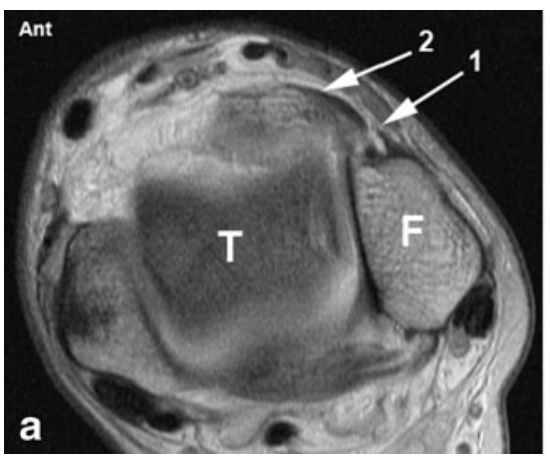

Fig. 3 Axial (a) and oblique (b) proton density TSE (turbo spin echo) images. In the axial plane (a), the ATIFL ( 1 ) is discontinuous. There is a large avulsion fragment from the anterior tibial tubercle (2). In the oblique

\section{Discussion}

Our study population consisted of 44 consecutive patients with an acute ankle fracture. The fracture distribution was in keeping with other studies with reported fracture rates by Lauge-Hansen type as follows: SE $42-72 \%$, PE 7-22\%, SA 6-20\%, and PA 5-21\% [25].

Many clinical and radiographic tests have been suggested to diagnose a disruption of the syndesmosis. Clinical tests include the squeeze test, Cotton test, exorotation stress test, and fibula translation test, but these are not reliable when syndesmotic injury is associated with an ankle fracture because of the pain, swelling, and dislocation [26]. Radiologically the syndesmosis can be evaluated with a radiograph, ultrasound, CT, or MRI. Several authors have argued that a radiograph has limited value in the assessment of syndesmotic integrity [27-29]. Stress radiographs with the foot in external rotation may demonstrate the diastasis but is rarely used in clinical practice [30]. Ultrasound can clearly depict a torn tibiofibular ligament in the acute stage but is less accurate after time has elapsed, and the results are dependent on the skills of the radiologist [31]. With the use of CT, widening of the mortise exceeding $3 \mathrm{~mm}$ can reliably be assessed, as well as gross deformity or malreduction [32-34].

The value of MRI in acute and chronic syndesmotic injuries has been described in several articles. Vogl et al.

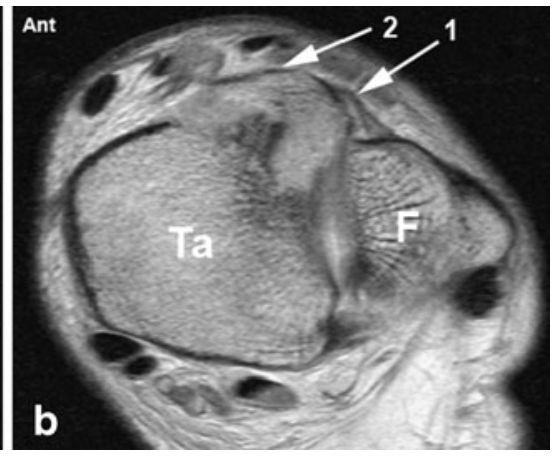

plane (b), the intact ATIFL is attached to the tibial avulsion fracture. This is an example of a Wagstaffe type I fracture (or Chaput-Tillaux fracture) with an intact ATIFL. $T$ Tibia, $F$ fibula, $T a$ talus, Ant anterior 
Fig. 4 Axial, oblique, and sagittal proton density TSE (turbo spin echo) images. In the axial plane (upper left), the ATIFL ( 1 ) is discontinuous. In front of the fibula lies an avulsed fibular fragment (2). In the oblique plane (lower left), the continuous ATIFL ( 1 ) is attached to the fibular avulsion (2). On the sagittal image (right), the avulsion fracture from the anterior tubercle of the fibula is just visible above the obliquely running fibula fracture. This is a type II Wagstaffe fracture: a fibular avulsion fragment attached to an intact ATIFL. Posteriorly the PTIFL (3) is ruptured in the axial plane (upper left). In the oblique plane (lower left), the intact PTIFL (3) is attached to a slip of periost from the posterior malleolus (4). $T$ Tibia, $F$ fibula, Ant anterior
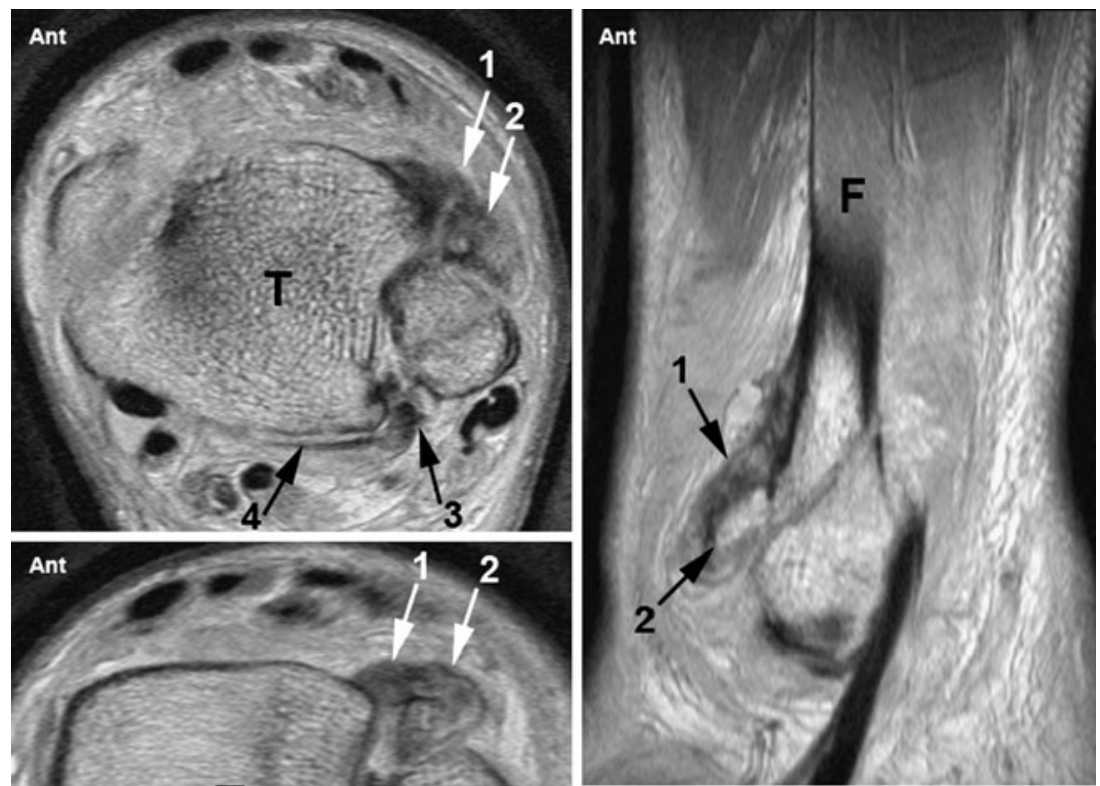

performed contrast-enhanced MRI in patients with acute ankle trauma and clinical suspicion of a syndesmotic tear, in which a tear of the ATIFL was markedly enhanced in T1weighted images [16]. Muratli et al. used MR arthrography in the first few days after injury to demonstrate leakage of contrast into the tibiofibular space as a sign of syndesmotic diastasis [35]. Schneck et al. paid attention to the position of the ligaments paraxial to the imaging plane and concluded that axial imaging with the foot in full dorsiflexion provided optimal views of the anterior and posterior tibiofibular ligaments [17].

However, imaging in the axial plane can result in falsepositive findings $[14,18]$ as the anterior and posterior distal tibiofibular ligaments run in an oblique plane $[19,20]$. Several studies reported that the most commonly injured and reliably visualized anterior tibiofibular ligament on MRI might not be seen in its entire length on one single transverse image and a partially imaged ligament may be mistaken for a tear despite careful observation of contiguous slices [16, 21, $22,24]$. Boonthathip et al. showed in cadavers that oblique image planes parallel to the long axis of the ligament better display the normal anatomy of the tibiofibular syndesmotic ligaments when compared with standard imaging planes [36]. Hermans et al. demonstrated in a recent study in healthy volunteers that the ATIFL and PTIFL are better depicted and that the integrity of the ligaments can be better evaluated in an oblique image plane of about $45^{\circ}$ than in the axial plane [23]. We therefore imaged the ATIFL and PTIFL with an additional $45^{\circ}$ oblique plane and compared this with the commonly used axial plane.

The interobserver agreement $(\mathrm{K})$ and agreement score [AS (\%)] regarding injury of the ATIFL and PTIFL were
Fig. 5 Axial (a) and oblique (b) proton density TSE (turbo spin echo) image. a Posterolateral oblique fracture of the posterior malleolus (1) with a discontinuous PTIFL (2) in the axial plane, but with an intact PTIFL in the oblique plane (b). In both planes, the ATIFL (3) is ruptured. $T$ Tibia, Ant anterior
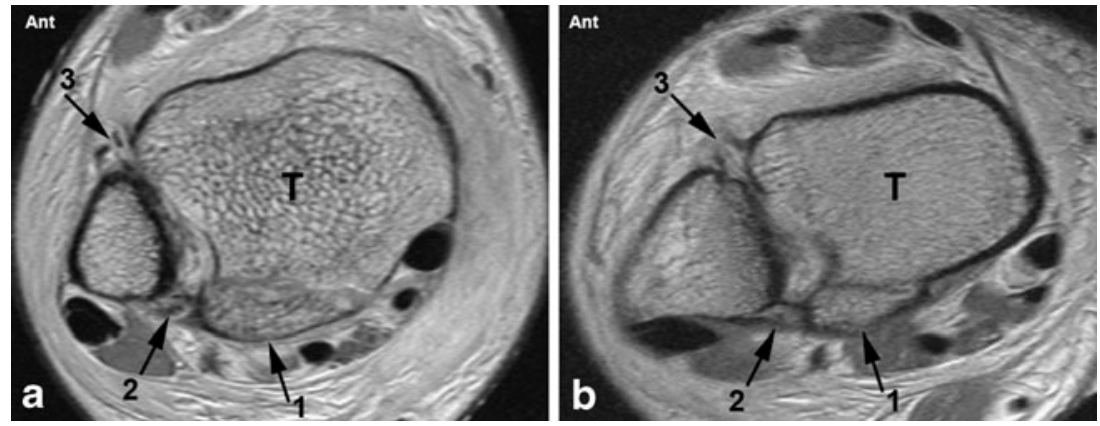
Table 3 Anterior and posterior overall syndesmotic injury on MRI, i. e., injury to distal tibiofibular ligament or avulsion fracture, in the axial (Ax) and oblique (Obl) image planes, related to the LaugeHansen (LH) fracture classification on the radiograph. Normal and thickened ligament $(0 / 1)$ and partial and ruptured ligament $(2 / 3)$ are grouped together

\begin{tabular}{|c|c|c|c|c|c|c|c|c|}
\hline \multirow[t]{3}{*}{ MRI } & \multicolumn{4}{|c|}{ LH anterior injury } & \multicolumn{4}{|c|}{ LH posterior injury } \\
\hline & \multicolumn{2}{|c|}{ Absent } & \multicolumn{2}{|c|}{ Present } & \multicolumn{2}{|c|}{ Absent } & \multicolumn{2}{|c|}{ Present } \\
\hline & Ax & Obl & $\mathrm{Ax}$ & Obl & Ax & Obl & $\mathrm{Ax}$ & Obl \\
\hline $0 / 1$ & 1 & 12 & 0 & 2 & 14 & 25 & 2 & 4 \\
\hline $2 / 3$ & 13 & 2 & 30 & 28 & 15 & 4 & 13 & 11 \\
\hline
\end{tabular}

good to excellent in both the axial and oblique image plane ( $\mathrm{k} 0.61-0.92$, AS $84-91 \%$ ). For both ligaments the oblique image plane demonstrated significantly lower injury scores than the axial plane $(p<0.001)$. In a recent study of Hermans et al. in 20 healthy volunteers, as well as in this study with 44 patients with an acute ankle fracture, the oblique image plane resulted in fewer false-positive findings.

The $k$ and AS regarding the presence of a fibular or tibial avulsion fracture were good to excellent in both the axial and oblique image planes ( $\mathrm{K} 0.71-0.84$, AS 91-95\%). There was no significant difference in detection of an avulsion fracture in the axial or oblique planes, neither anteriorly $(p=0.50)$ nor posteriorly $(p=1.00)$. In the axial plane in $2 / 3$ patients with a tibial avulsion and $5 / 6$ patients with a fibular avulsion, injury of the ATIFL was detected, whereas in the oblique plane the bony avulsion was present without a rupture of the ATIFL.

In 1875 Wagstaffe was the first to describe two cases of an avulsion fracture from the anterior tibiofibular ligament [37]. In case of a Wagstaffe fracture combined with a lateral malleolar fracture, the ATIFL avulsion fragment was classified as type I, II, or III. Type I is a displaced avulsion fracture of the distal end of the tibia and is very rare. The most commonly occurring type is type II, which is a fracture of the anterior spike of the proximal fibular fragment. Type III is a fracture of the anterior tubercles from both the tibia and fibula and is also very rare [38, 39]. In our study we found $7 / 44$ patients with a type II Wagstaffe fracture on MRI but not on the radiograph. If the surgeon can reduce the avulsed fragment properly and obtain stable fixation, direct bone-to-bone union can be achieved, inducing more physiologic healing of the syndesmosis. Park et al. described one case in which the Wagstaffe fracture was displaced into the joint and caused chronic ankle pain and restricted joint motion [38]. Nelson described $18 \%$ fibular avulsions (Wagstaffe type II fractures) and $8 \%$ tibial avulsions (Wagstaffe type I or ChaputTillaux fractures) in a series of 50 transmalleolar fractures. In some cases the avulsed fragment did wedge the joint open [9]. A Wagstaffe fracture can be easily overlooked because of a fracture hematoma or torn capsule or if it is
Fig. 6 Axial (upper left) and oblique (lower left) proton density TSE (turbo spin echo) image. AP (upper right) and lateral (lower right) radiograph. A local swelling at the level of the distal fibula is visible on the AP radiograph. The lateral radiograph shows an oblique fracture in the distal fibula. According to Lauge-Hansen this is a supination-external rotation type 2 fracture (SE2). In the axial plane (upper left), the ATIFL (1) and PTIFL (2) are both discontinuous. In the oblique plane (lower left), the ATIFL is also discontinuous, but the multifascicular PTIFL is intact. The fracture line through the fibula is visible in both planes. $T$ Tibia, Ant anterior
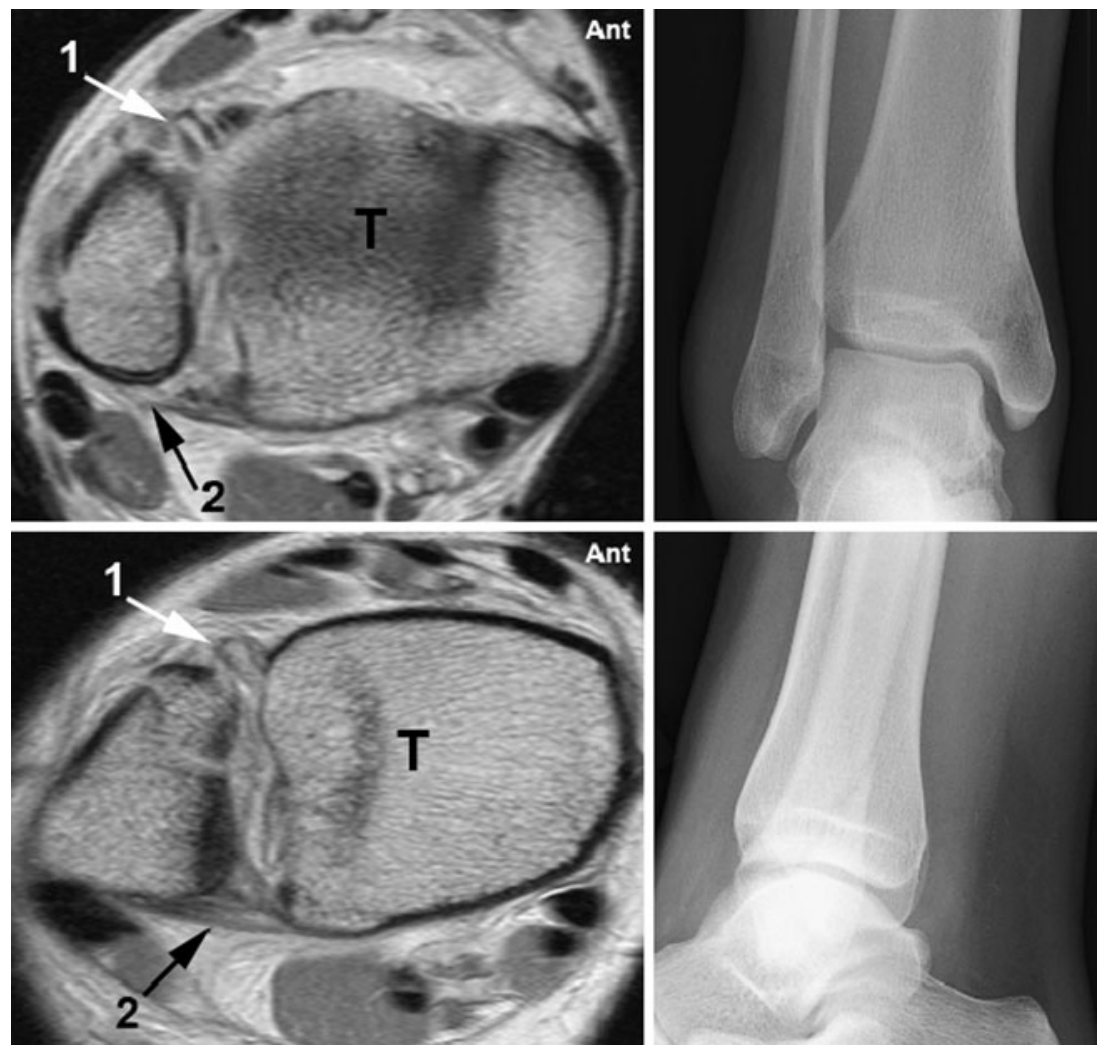
covered by an intact extensor retinaculum. Therefore careful examination of the anterior syndesmosis area is necessary after fixation of the lateral malleolar fracture.

Posteriorly we only found avulsion fractures of the tibia and no avulsion fractures of the fibula. In 6/11 patients with a tibial avulsion, the PTIFL appeared to be ruptured in the axial plane and was shown to be intact in the oblique plane. Generally speaking, when an avulsion fracture occurs, the ligament attached to the avulsion remains intact. Therefore our findings in the oblique image plane were concordant with this generally accepted statement. This finding has important implications for treatment of unstable ankle fractures. In case of a fracture of the distal posterior tibia with an intact PTIFL, syndesmotic stability can be achieved by fixation of the posterior malleolus fragment without the use of a syndesmotic screw [40]. An additional value of MRI is information about the exact size, location, and orientation of the posterior malleolus fracture. This may help the surgeon in choosing the surgical approach to the fragment [41].

Injury of the anterior or posterior distal tibiofibular syndesmosis can be either a rupture of the distal tibiofibular ligament or a bony avulsion from either the fibula or tibia. The $k$ value regarding injury of the anterior syndesmosis was moderate $(K=0.48)$ for the axial plane and very good $(\kappa=0.81)$ for the oblique plane, although the AS for both planes was good ( 84 and $86 \%$, respectively). Discrepancy between the percentage agreement and $k$ value can be explained by the skewed distribution of categories in our study. In these cases, $K$ is known to have poor properties as an index for measuring agreement [42]. The $k$ and AS for posterior syndesmosis injury were very good.

To further substantiate our data, we compared our findings at MRI with the Lauge-Hansen fracture classification, as with this classification it is possible to predict injury to the anterior and posterior syndesmosis. For the oblique MRI plane, the specificity increased for both anterior (to $86 \%$ from $7 \%$ ) and posterior (to $86 \%$ from $48 \%$ ) syndesmotic injury when compared to the axial plane. This means a substantial decrease in false-positive diagnoses of the ATIFL and PTIFL. Although we do not know if the Lauge-Hansen fracture classification allows an anatomically correct diagnosis, it is likely that the oblique images are closer to the diagnosis than the axial images.

Several studies focused on the position of the foot during imaging in order to evaluate the ankle ligaments. The best full-length visualization of the ATIFL and PTIFL would be obtained in the axial plane, with the foot taped into full dorsiflexion $[15,17]$. As it is difficult to obtain a position of full dorsiflexion in a patient with an acute ankle fracture, we maintained the foot in a neutral position. It is hard to imagine what influence the foot in dorsiflexion would have on imaging in the axial plane of the obliquely running ATIFL and PTIFL. With dorsiflexion the only effect on the ligaments would be an increased tension, as the talus pushes the fibula outwards. It therefore seems more likely that the angle of the image plane rather than the position of the foot plays a more important role in depicting the syndesmotic ligaments. This concept is confirmed in the current study, in which the oblique plane showed a decrease in false-positive findings, especially regarding injury of the ATIFL. This finding is the more relevant as, until now, the ATIFL was the most reliably visualized syndesmotic ligament in an axial MRI. Thus, MRI could help in selecting patients for an open reduction with internal fixation (ORIF) in case of suspicion of syndesmotic injury in an acute ankle fracture.

One shortcoming of our study was that, due to logistical problems, it was not possible to obtain a preoperative MRI in $5 / 44$ of patients. However, in the patients with a plate and screws, the quality of the MR image was still good, and the ATIFL and PTIFL as well as the presence of a tibial or fibular avulsion could be well evaluated (Fig. 7). Secondly, we did not have an invasive technique, such as arthroscopy, available to confirm our MRI findings. However, in our opinion it would not be ethical to perform an ankle arthroscopy in patients who could be treated with only a plaster. Moreover, our study population now comprises both patients with and without syndesmotic injury, which therefore reduces the selection bias that would occur if only
Fig. 7 Axial (a) and oblique (b) proton density TSE (turbo spin echo) images. Posterior tibial periost slip (1) with a discontinuous PTIFL (2) in the axial plane (a) and an intact PTIFL in the oblique plane (b). In both planes the ATIFL (3) is ruptured. Although there is a screw present in the fibula (4), the quality of the MR image is still good and the tibiofibular ligaments can be well evaluated. $T$ Tibia, $F$ fibula, Ant anterior
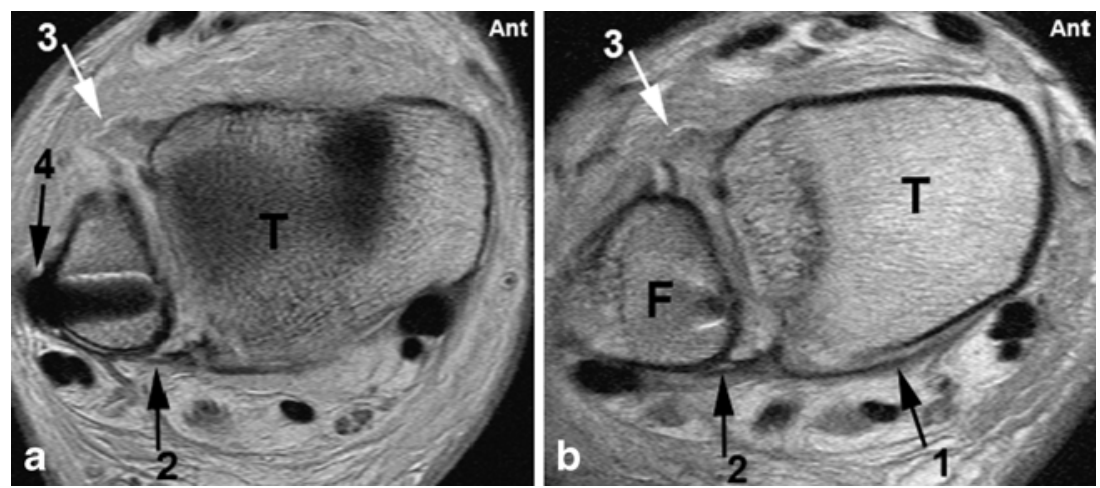
operated patients were included. In the latter case, the a priori chance of syndesmotic injury would have been very high.

In conclusion, our results show the additional value of an oblique MR image plane for detection of injury of the anterior and posterior distal tibiofibular syndesmoses in acute ankle fractures. More accurate information about the tibiofibular syndesmosis could lead to a better treatment plan, resulting in fewer complaints of chronic syndesmotic injury or even early osteoarthritis.

\section{Conclusion}

Our results show the additional value of an oblique MR image plane for detection of injury of the anterior and posterior distal tibiofibular syndesmosis in acute ankle fractures. The $45^{\circ}$ oblique MRI plane demonstrated significantly less ligamentous injury than the axial planes, for both the ATIFL and PTIFL. Findings of syndesmotic injury in the oblique MRI plane were closer to the diagnosis as assumed based on the Lauge-Hansen classification than in the axial plane. With more accurate information, the surgeon can better decide when to stabilize syndesmotic injury in acute ankle fractures.

Conflict of interest The authors declare that they have no conflict of interest.

Open Access This article is distributed under the terms of the Creative Commons Attribution Noncommercial License which permits any noncommercial use, distribution, and reproduction in any medium, provided the original author(s) and source are credited.

\section{References}

1. Kennedy JG, Johnson SM, Collins AL, DalloVedova P, McManus WF, Hynes DM, et al. An evaluation of the Weber classification of ankle fractures. Injury. 1998;29(8):577-80.

2. Court-Brown CM, McBirnie J, Wilson G. Adult ankle fracturesan increasing problem? Acta Orthop Scand. 1998;69(1):43-7.

3. Lindsjo U. Operative treatment of ankle fractures. Acta Orthop Scand Suppl. 1981;189:1-131.

4. Pettrone FA, Gail M, Pee D, Fitzpatrick T, Van Herpe LB. Quantitative criteria for prediction of the results after displaced fracture of the ankle. J Bone Joint Surg Am. 1983;65(5):667-77.

5. Yamaguchi K, Martin CH, Boden SD, Labropoulos PA. Operative treatment of syndesmotic disruptions without use of a syndesmotic screw: a prospective clinical study. Foot Ankle Int. 1994;15 (8):407-14.

6. Weening B, Bhandari M. Predictors of functional outcome following transsyndesmotic screw fixation of ankle fractures. $\mathrm{J}$ Orthop Trauma. 2005;19(2):102-8.
7. Ramsey PL, Hamilton W. Changes in tibiotalar area of contact caused by lateral talar shift. J Bone Joint Surg Am. 1976;58 (3):356-7.

8. Cedell CA. Total rupture of the syndesmosis in the ankle joint. Lakartidningen. 1969;66(23):2427-33.

9. Nelson OA. Examination and repair of the AITFL in transmalleolar fractures. J Orthop Trauma. 2006;20(9):637-43.

10. Hovis WD, Kaiser BW, Watson JT, Bucholz RW. Treatment of syndesmotic disruptions of the ankle with bioabsorbable screw fixation. J Bone Joint Surg Am. 2002;84A(1):26-31.

11. Kennedy JG, Soffe KE, Dalla Vedova P, Stephens MM, O'Brien T, Walsh MG, et al. Evaluation of the syndesmotic screw in low Weber $\mathrm{C}$ ankle fractures. J Orthop Trauma. 2000;14(5):359-66.

12. Chissell HR, Jones J. The influence of a diastasis screw on the outcome of Weber type-C ankle fractures. J Bone Joint Surg Br. 1995;77(3):435-8.

13. Brown KW, Morrison WB, Schweitzer ME, Parellada JA, Nothnagel H. MRI findings associated with distal tibiofibular syndesmosis injury. AJR Am J Roentgenol. 2004;182(1):1316.

14. Takao M, Ochi M, Oae K, Naito K, Uchio Y. Diagnosis of a tear of the tibiofibular syndesmosis. The role of arthroscopy of the ankle. J Bone Joint Surg. 2003;85(3):324-9.

15. Muhle C, Frank LR, Rand T, Ahn JM, Yeh LR, Trudell D, et al. Tibiofibular syndesmosis: high-resolution MRI using a local gradient coil. J Comput Assist Tomogr. 1998;22(6):93844.

16. Vogl TJ, Hochmuth K, Diebold T, Lubrich J, Hofmann R, Stockle $\mathrm{U}$, et al. Magnetic resonance imaging in the diagnosis of acute injured distal tibiofibular syndesmosis. Invest Radiol. 1997;32 (7):401-9.

17. Schneck CD, Mesgarzadeh M, Bonakdarpour A, Ross GJ. MR imaging of the most commonly injured ankle ligaments. Part I. Normal anatomy. Radiology. 1992;184(2):499-506.

18. Oae K, Takao M, Naito K, Uchio Y, Kono T, Ishida J, et al. Injury of the tibiofibular syndesmosis: value of MR imaging for diagnosis. Radiology. 2003;227(1):155-61.

19. Bartonicek J. Anatomy of the tibiofibular syndesmosis and its clinical relevance. Surg Radiol Anat. 2003;25(5-6):379-86.

20. Hermans JJ, Beumer A, De Jong AW et al. Anatomy of the distal tibiofibular syndesmosis in adults: a pictorial essay with a multimodality approach. J Anat. 2010;217(6):633-45.

21. Han SH, Lee JW, Kim S, Suh JS, Choi YR. Chronic tibiofibular syndesmosis injury: the diagnostic efficiency of magnetic resonance imaging and comparative analysis of operative treatment. Foot Ankle Int. 2007;28(3):336-42.

22. Lee SH, Jacobson J, Trudell D, Resnick D. Ligaments of the ankle: normal anatomy with MR arthrography. J Comput Assist Tomogr. 1998;22(5):807-13.

23. Hermans JJ, Ginai AZ, Wentink $\mathrm{N}$ et al. The additional value of an oblique image plane for MRI of the anterior and posterior distal tibiofibular syndesmosis. Skeletal Radiol. 2011;40(1):75-83.

24. Kim S, Huh YM, Song HT, Lee SA, Lee JW, Lee JE, et al. Chronic tibiofibular syndesmosis injury of ankle: evaluation with contrast-enhanced fat-suppressed 3D fast spoiled gradient-recalled acquisition in the steady state MR imaging. Radiology. 2007;242 (1):225-35.

25. Lindsjo U. Classification of ankle fractures: the Lauge-Hansen or AO system? Clin Orthop Relat Res. 1985;199:12-6.

26. van den Bekerom MP, Lamme B, Hogervorst M, Bolhuis HW. Which ankle fractures require syndesmotic stabilization? J Foot Ankle Surg. 2007;46(6):456-63.

27. Pneumaticos SG, Noble PC, Chatziioannou SN et al. The effects of rotation on radiographic evaluation of the tibiofibular syndesmosis. Foot Ankle Int. 2002;23(2):107-11. 
28. Beumer A, van Hemert WL, Niesing R, Entius CA, Ginai AZ, Mulder PG, et al. Radiographic measurement of the distal tibiofibular syndesmosis has limited use. Clin Orthop Relat Res. 2004;423:227-34.

29. Brage ME, Bennett CR, Whitehurst JB et al. Observer reliability in ankle radiographic measurements. Foot Ankle Int. 1997;18 (6):324-9.

30. Dattani R, Patnaik S, Kantak A, Srikanth B, Selvan TP. Injuries to the tibiofibular syndesmosis. J Bone Joint Surg Br. 2008;90(4):405-10.

31. Milz P, Milz S, Steinborn M, Mittlmeier T, Putz R, Reiser M. Lateral ankle ligaments and tibiofibular syndesmosis. $13-\mathrm{MHz}$ high-frequency sonography and MRI compared in 20 patients. Acta Orthop Scand. 1998;69(1):51-5.

32. Ebraheim NA, Lu J, Yang $\mathrm{H}$ et al. Radiographic and CT evaluation of tibiofibular syndesmotic diastasis: a cadaver study. Foot Ankle Int. 1997;18(11):693-8.

33. Elgafy H, Semaan HB, Blessinger B, Wassef A, Ebraheim NA. Computed tomography of normal distal tibiofibular syndesmosis. Skeletal Radiol. 2010;39(6):559-64.

34. Gardner MJ, Demetrakopoulos D, Briggs SM, Helfet DL, Lorich DG. Malreduction of the tibiofibular syndesmosis in ankle fractures. Foot Ankle Int. 2006;27(10):788-92.
35. Muratli HH, Bicimoglu A, Celebi L, Boyacigil S, Damgaci L, Tabak AY. Magnetic resonance arthrographic evaluation of syndesmotic diastasis in ankle fractures. Arch Orthop Trauma Surg. 2005;125(4):222-7.

36. Boonthathip M, Chen L, Trudell DJ, Resnick DL. Tibiofibular syndesmotic ligaments: MR arthrography in cadavers with anatomic correlation. Radiology. 2010;254(3):827-36.

37. Wagstaffe W. An unusual form of fracture of the fibula. Cited in: St Thomas Hosp Rep.1875;6:43.

38. Park JW, Kim SK, Hong JS, Park JH. Anterior tibiofibular ligament avulsion fracture in weber type B lateral malleolar fracture. J Trauma. 2002;52(4):655-9.

39. Pankovich AM. Fractures of the fibula at the distal tibiofibular syndesmosis. Clin Orthop Relat Res. 1979;143:138-47.

40. Gardner MJ, Brodsky A, Briggs SM, Nielson JH, Lorich DG. Fixation of posterior malleolar fractures provides greater syndesmotic stability. Clin Orthop Relat Res. 2006;447:165-71.

41. Haraguchi N, Haruyama H, Toga H, Kato F. Pathoanatomy of posterior malleolar fractures of the ankle. J Bone Joint Surg Am. 2006;88(5):1085-92.

42. Feinstein AR, Cicchetti DV. High agreement but low kappa: I. The problems of two paradoxes. J Clin Epidemiol. 1990;43(6):543-9. 